\begin{tabular}{|l|l|l|l|l|}
\hline Revista Clío América & ISSN: 1909-941X & Vol. 8 & No. 15 & Enero - Junio de 2014 \\
\hline
\end{tabular}

\title{
Ocultamiento del discurso pedagógico frente al discurso administrativo en la gestión de las organizaciones educativas
}

\author{
Teaching speech to the hidden discourse of administration in the management of \\ educational organizations
}

\begin{abstract}
Resumen: Este artículo presenta parte de los resultados finales del Proyecto de Investigación titulado: "TENSIONES ENTRE LOS DISCURSOS PEDAGÓGICOS Y LOS ADMINISTRATIVOS, A PARTIR DE LA IMPLEMENTACIÓN DE LOS SISTEMAS DE GESTIÓN DE CALIDAD EN LAS INSTITUCIONES DE EDUCACIÓN BÁSICA Y MEDIA: Una lectura evaluativa y propositiva". Como conclusión central se establece y argumenta que los discursos pedagógicos están siendo desplazados por los discursos administrativos en las instituciones educativas; a partir de la implementación de diferentes Sistemas de Gestión de Calidad. Este desplazamiento está ocasionando que la identidad de las organizaciones educativas se vea afectada y que el eje de la actividad cotidiana de docentes y directivos se centre más en la gestión administrativa de la organización, que en el desarrollo de sus prácticas pedagógicas. Debido a la intencionalidad que orientó esta investigación, la naturaleza del objeto de estudio y en coherencia con su complejidad social, ésta se concibió, en lo fundamental, bajo un enfoque cualitativo; apuntando a un esfuerzo por comprender la realidad social alusiva a las tensiones que se están sucediendo entre los discursos pedagógicos y administrativos a partir de la implementación de los sistemas de gestión de la calidad en las instituciones de educación básica y media.
\end{abstract}

Palabras clave: Discurso Pedagógico; Discursos Administrativo; Organizaciones Educativas; Gestión Educativa; Calidad Educativa.

JEL: H52, I21, I23

Abstract: This article presents part of the final results of the research project entitled: "TENSIONS BETWEEN TEACHING STATEMENTS AND ADMINISTRATIVE FROM THE IMPLEMENTATION OF SYSTEMS OF QUALITY MANAGEMENT IN INSTITUTIONS OF BASIC EDUCATION AND SECONDARY: An evaluative and purposeful reading". A central conclusion is established and argues that pedagogical discourses are being displaced by the administrative discourses in educational institutions; from the implementation of different quality management systems. This shift is causing identity of educational organizations is affected and that the axis of the daily activities of teachers and administrators to focus more on the administrative management of the organization in the development of their teaching. Because the intent that guided this research, the nature of the object of study and in line with its social complexity, it was designed, essentially, under a qualitative approach; pointing to an effort to understand the

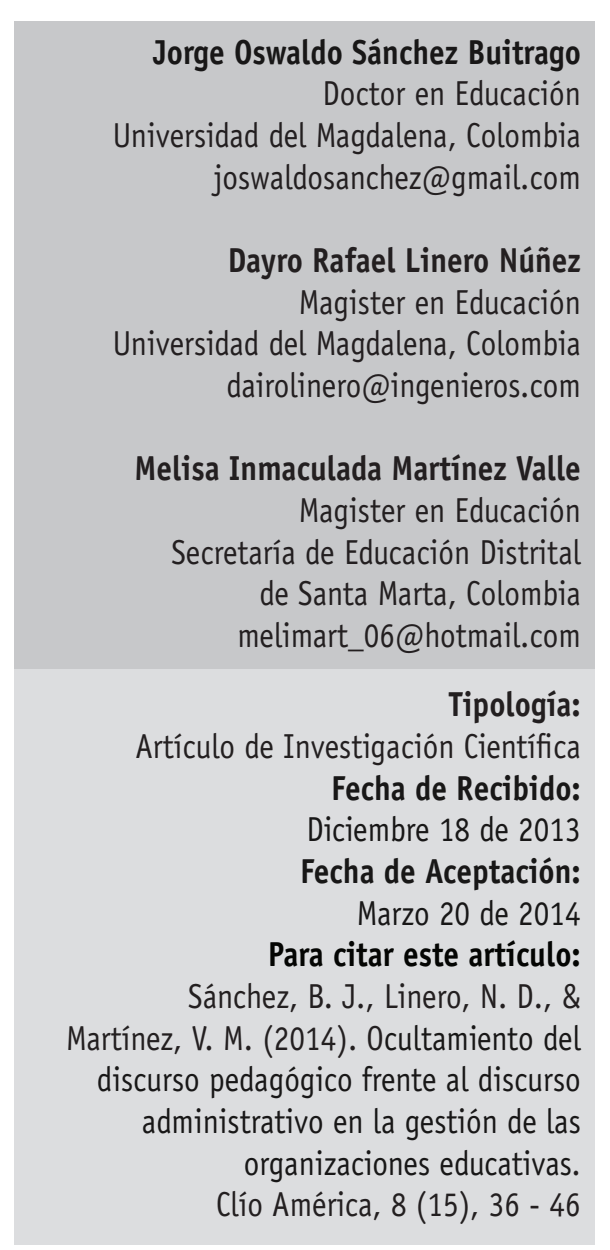


social reality alluding to the tensions that are happening between the pedagogical and administrative from the implementation of systems quality management in primary and secondary education institutions.

Key words: Speech Teaching, Administrative Speeches, Educational Organizations, Educational Management, Educational Quality.

\section{Introducción}

En los últimos años se ha dado un auge importante de intervención y mejoramiento de los procesos de gestión de la calidad al interior de las instituciones educativas, a partir de dos modelos básicos: EFQM e ISO. Este auge ha determinado en estas instituciones la irrupción de lenguajes y prácticas de gestión derivadas de los Sistemas de Gestión de Calidad (SGC). El uso de la categoría "irrupción", se hace para denotar que los Sistemas de Gestión de Calidad han llegado al escenario de la gestión y el desarrollo de las instituciones educativas de manera intempestiva. Estas irrupciones han provocado tensiones discursivas y operativas entre la racionalidad pedagógica y la administrativa en la gestión de las organizaciones educativas; las cuales no han sido lo suficientemente exploradas y mucho menos teorizadas y documentadas.

En esta investigación la categoría tensión fue tratada desde la perspectiva planteada por Schvarstein, el cual afirma;

la tensión no es una mala palabra; si hablamos de los nuevos paradigmas en gestión humana, el conflicto, la tensión y la contradicción en el sentido más puro de la palabra son el "motor" de la organización, lo que genera su dinámica, lo que abre la posibilidad para que evolucione y cambie. Las organizaciones que dicen "no tener conflictos" son organizaciones totalitarias porque han impuesto una de las dos lógicas por sobre la otra. (2008, pág. 3)

Esta investigación, se desarrolló desde dos perspectivas, la primera es desde el punto de vista epistémico, la cual consistió en explorar, dilucidar y argumentar teóricamente las tensiones que se están sucediendo entre los discursos y prácticas pedagógicas y los discursos y prácticas administrativas; a partir de la implementación de los Sistemas de Gestión de Calidad en las instituciones de educación básica y media, soportados en los modelos de gestión de calidad EFQM e ISO 9000, validados por el MEN. Un Sistema de Gestión de la Calidad es una forma de trabajar, mediante la cual una organización asegura la satisfacción de las necesidades de sus clientes. Por lo que tiene que planificar, mantener y mejorar continuamente el desempeño de sus procesos, bajo un esquema de eficiencia y eficacia. EFQM trata de un modelo de gestión de la calidad no normativo, cuyo concepto fundamental es la autoevaluación basada en un análisis detallado del funcionamiento del Sistema de Gestión de la organización usando como guía los criterios del modelo, identificando los puntos fuertes y las áreas de mejora, a partir de las cuales se desarrollan acciones tendientes a la mejora de las mismas. Mientras que por medio de un SGC bajo La Norma ISO 9000 la calidad se busca bajo la luz de 8 principios que son utilizados para dirigir y operar una organización con éxito y para tal fin promueve una gestión sistemática y visible.

Por otra parte, desde el punto de vista empírico, la investigación se orientó a valorar el impacto de las prácticas y discursos administrativos, provenientes de los SGC, en las prácticas y discursos pedagógicos de las instituciones. Entendiendo los discursos administrativos como

una tradición, tanto por ser un conjunto de conceptos, de teorías, interpretaciones, normas, prácticas, valores y estándares de evaluación en el ámbito de acción de las organizaciones y del conocimiento administrativo: como por haber tenido continuidad en el tiempo, enriqueciéndose, llegando a tener arraigo histórico y autoridad para orientar y evaluar las creencias y prácticas de sus adherentes. (Galicia, 2009, pág. 139) 
Por su parte, en el "discurso pedagógico" se destaca la importancia de la comunicación, que permite la transmisión de una historia que poco a poco se va construyendo y apoya a todo un contexto a adquirir herramientas que faciliten la labor de la enseñanza, de los contenidos en sí y de unas prácticas pedagógicas que permite regular la producción, reproducción, y los cambios de las acciones educativas, sus relaciones sociales de transmisión, adquisición y la organización de sus contextos (Bernstein, 2003)

Fue entonces desde el punto de vista teórico, una investigación centrada en el esclarecimiento de las tensiones existentes entre la naturaleza del discurso pedagógico y la naturaleza del discurso administrativo, así como en la latente multiplicidad de concepciones sobre lo pedagógico y lo administrativo. Mientras que desde lo empírico, esta investigación permitió hacer evidente el ocultamiento práctico del discurso pedagógico como orientador y regulador de la actividad académica de los profesionales de la educación; cediendo la conducción teórica a los lenguajes derivados y asociados con los SGC. Categorías como "mejoramiento continuo", "no conformidades", "conformidades", "hallazgos", "producto conforme", "producto no conforme", "ciclo de la calidad", etc., están irrumpiendo en la cotidianidad de los espacios reflexivos de los educadores y gestores educativos en sus instituciones educativas y están desplazando saberes específicos de su quehacer pedagógico; es decir se están posicionando en sus prácticas discursos "despedagogizados". Los hallazgos empíricos que sustentan esta postura son precisamente los resultados que se presentan en este artículo. dado a lo extensivo de los resultados obtenidos en la investigacón, ha sido necesario abordarlos en otro artículo.

Los hallazgos encontrados en esta investigación se han producido con la ayuda exploratoria de los siguientes interrogantes investigativos:

- ¿Cuáles son los constituyentes primarios que configuran la naturaleza de las categorías "discurso pedagógico" y "discurso administrativo" y permiten su delimitación teórica?

- ¿Cuáles son las tensiones específicas que se están reconociendo entre las categorías "discurso pedagógico" y "discurso administrativo", cuando estas confluyen en el ámbito argumentativo de la gestión de las instituciones educativas?

- ¿En las Instituciones Educativas, las prácticas y discursos pedagógicos están siendo desterritorializados por las prácticas y discursos administrativos propios de los SGC?

A partir de los problemas enunciados, la investigación se centró en la siguiente pregunta generadora central: ¿Cuáles son las tensiones entre las categorías "discursos y prácticas pedagógicas" y "discursos y prácticas administrativas", derivadas de la implementación de los SGC en las instituciones de educación básica y media de la ciudad de santa marta?

En este contexto de sentido esta investigación se orientó en función del siguiente objetivo: Argumentar teóricamente las tensiones entre las categorías "discursos y prácticas pedagógicas" y "discursos y prácticas administrativas", derivadas de la implementación de los SGC en las instituciones de educación básica y media de la ciudad de santa marta.

\section{Metodología}

En este apartado se presenta el enfoque investigativo así como la manera en cómo fue recopilada, procesada y analizada la información de la investigación.

En el proceso se contempló la perspectiva del enfoque cualitativo planteado por Deslauriers, cuando afirma que

la investigación cualitativa no rechaza las cifras ni las estadísticas pero no les concede simplemente el primer lugar; se concentra ante todo sobre el análisis de los procesos sociales, sobre el sentido que las personas y los colectivos dan a la acción, sobre la vida cotidiana, sobre la construcción de la realidad social. (2004, pág. 6)

La información se recopiló en las tres instituciones educativas que hicieron parte del estudio de casos múltiples de la investigación. En cada una de las instituciones se realizó una entrevista con grupo focal y tres entrevistas en profundidad a tres actores de cada institución, de donde surgió el material 
que se transcribió y nutrió el discurso oral. De igual forma se revisaron las actas del consejo directivo, del consejo académico, del comité de calidad, el texto formal del PEI y del Manual de Calidad; como expresiones del discurso escrito. Toda esta información se sistematizó y analizó con la ayuda del Software Atlas-Ti, versión 6.0 (Software utilizado en investigaciones de naturaleza cualitativa); es preciso aclarar que en este artículo sólo se presenta parte de los resultados obtenidos en la investigación, debido a lo extenso de la información existente.

La forma como fue procesada la información sucedió así: en un primer momento, se configuró una estructura de categorías y subcategorías, trabajadas jerárquicamente a nivel escrito y oral, a través de esquemas o mapas conceptuales (ver más adelante en Figura 2.), los cuales se agruparon dentro de dos categorías: Discurso escrito (fuentes instituidas) y discurso oral (fuentes instituyentes). Con la ayuda del Software mencionado, fueron identificadas y asignadas Unidades Semánticas (US) a cada una de las categorías correspondientes. US, hace referencia al "significado de diversas palabras que pueden descomponerse en unidades mínimas de significación semántica o semas" (Ducrot \& Todorov, 1945, pág. 206). Las Unidades Semánticas se trabajan en el presente proyecto, como el conjunto de significaciones relacionadas con un determinado contexto 0 con una determinada situación del discurso.

Una vez culminada la labor de procesamiento de la información recopilada, se procedió a establecer el número de US pertenecientes a las categorías propias de cada uno de los discursos antes mencionados (Discursos Pedagógicos y Discursos Administrativos), estas cantidades fueron tabuladas y luego llevadas a graficas de frecuencia, los cuales sirvieron de soporte para el análisis que pone de manifiesto la desterritorialización del Discurso Pedagógico por el Discurso Administrativo al interior de las instituciones educativas.

\section{Resultados y Discusión}

La presente investigación, desde sus inicios contempló la siguiente Hipótesis: La irrupción de los discursos administrativos en las I.E., a partir de la implementación de los Sistemas de Gestión de
Calidad -SGC-, está ocasionando el desplazamiento del discurso pedagógico y el posicionamiento instrumental del discurso administrativo.

Para la comprobación de la hipótesis en mención, en esta investigación, con la ayuda del Software Atlas ti, se procesó toda la información recopilada en las instituciones participantes, esto es, aquellas provenientes tanto de fuentes instituidas como de fuentes instituyentes. Una vez hecho esto, se procedió a cuantificar el número de US, obteniendo los siguientes resultados:

El total de Unidades Semánticas encontradas tanto en fuentes instituida como instituyentes fue de 841 , distribuidas entre las categorías Discurso Pedagógico y Discurso Administrativo con 394 (47\%) y $447(53 \%)$ US respectivamente. Se confirmó que el Discurso Administrativo se encuentra desterritorializando al Discurso Pedagógico. Destacándose la importancia que se le da a aspectos como la "mejora continua" y la "planeación estratégica", los cuales hacen parte fundamental en los PEI de las Instituciones Educativas, así se encuentra referenciado en uno de ellos, donde se contempla que

"Implementar el modelo en la Institución
Educativa conlleva al trabajo en torno a cuatro
momentos importantísimos como son: La pla-
neación, la ejecución, el control y los ajustes
para la mejora continua Todo el proceso de la
gestión estratégica está acompañado de un
PLAN ESTRATÉGICO y de los PLANES DE MEJORA.
Además hay un control permanente en manos
del EQUIPO DE EXCELENCIA, ayUdado de un
excelente PLAN DE COMUNICACIONES". (PEI de
institución fOCal 1).

De igual forma en los manuales de calidad se encuentran muy marcados estos aspectos, destacándose en uno de ellos que

"La mejora continua se evidencia con las acciones de mejora efectivamente aplicadas, el control a los objetivos de calidad, los resultados de las auditorías internas, al desempeño a los procesos, acciones correctivas y preventivas y los resultados de la revisión por la Rectoría". (Manual de Calidad de institución focal 2) 
Así mismo, a partir de las entrevistas realizadas fue posible constatar que esa incursión de nuevas terminologías, ocasionó mucha resistencia sobre todo en el personal docente, esto lo ratifica una docente cuando afirma que:

" muchos de los profesores se resistieron, ya que cambia el modelo que venían trabajando, la oralidad, la cultura de la medición y el tener que incorporarse al discurso administrativo, al que fue necesario hacerlo, cuando antes la mayoría de los profesores de pronto desconocian esto, las cuatro áreas de gestión, los diecinueve procesos que están en la guía 34, los noventa y tanto procedimientos, más los que nosotros nos habiamos inventado, en fin, fue todo un conflicto con estas palabras que se utilizaban." (Docente de institución focal 2)

En la figura 1, se muestra el porcentaje total que obtuvo cada discurso tanto en su aspecto instituido como instituyente, evidenciándose una mayor recurrencia de US en el Discurso Administrativo (DA) que en el Discurso Pedagógico (DP), notándose la incidencia que ha tenido la administración, al momento de incursionar en el ámbito educativo a través de los SGC. Ya que como lo afirma un docente:

"Al momento en el que la directriz, la parte administrativa cambia, transforma ese discurso pedagógico, porque ya nos metieron la influencia de que era así y debiamos evaluarnos continuamente para ser mejores y excelentes cada día, es decir, la mejora continua. Al momento que sucede eso en la institución, hay una transformación en cada uno de los docentes, porque desde sus áreas de mejoras, desde su implementación teníamos la responsabilidad de contribuir en ese proceso y cumplir con los requerimientos de la norma" (Docente de institución focal 1).

\section{- Figura 1.}

\section{Peso total de discursos}

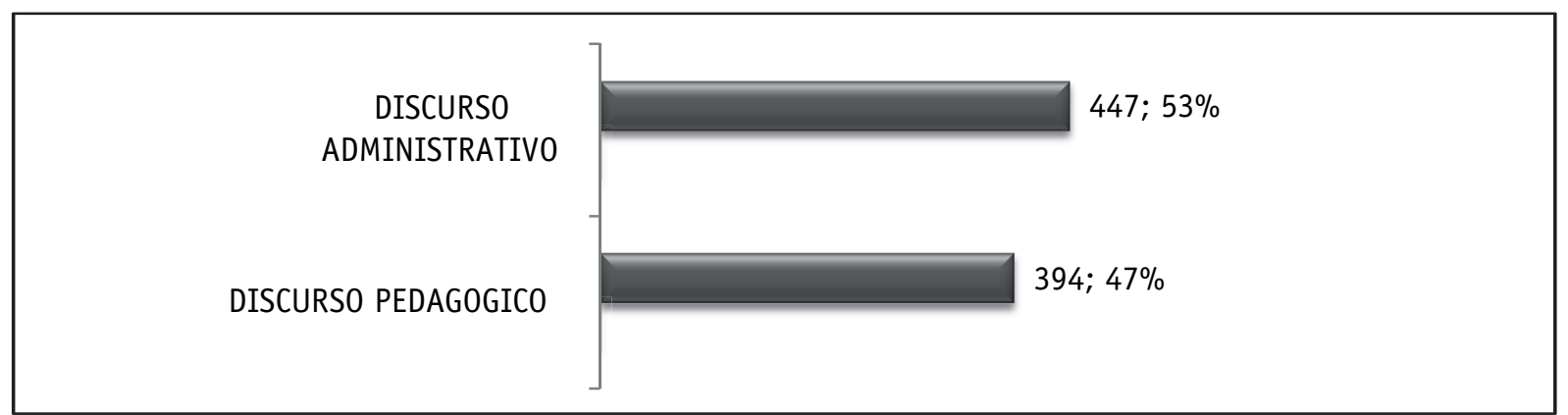

Fuente: Documentos (Actas, PEI, Manuales de calidad) y textos orales transcritos recopilados en talleres con grupos focales de discusión, y procesados en Software Atlas- ti. 
A continuación se aborda la descripción, análisis y reflexión correspondiente al Discurso Escrito y luego al Discurso Oral; categoría fuentes Instituidas $\mathrm{y}$ fuentes instituyentes respectivamente.

\section{Ocultamiento del Discurso pedagógico en Fuentes Instituidas}

Como se expresó inicialmente en la nota al pie de página, Según Schvarstein (1992), (citado en Belmonte \& Gadano, 2007, p.115), el Discurso Instituido hace referencia a "aquello que está establecido, es el conjunto de normas y valores dominantes así como el sistema de roles que constituyen el sostén de todo orden social". Se podría decir entonces, que "lo instituido", es el sistema de pautas y valores que orientan las conductas y funciones dentro de la Institución Educativa, es todo aquello que está estipulado y que sirve de guía, para que en este caso, los actores educativos, conozcan y vinculen a su quehacer diario dichos requerimientos.

Parafraseando a González, a este tipo de discursos, también es posible denominarlos, como el "discurso dominante", el cual sería todo aquello que se da por supuesto, que se encuentra arraigado y se considera incuestionable. Las instituciones, especialmente las educativas, juegan un papel protagónico, ya que mantienen la validez de los discursos y los transmiten. Las instituciones cobran cuerpo a partir de las normas que los constituyen, son ellas las encargadas de velar porque lo dado por supuesto se dé y se mantenga como tal. Lo instituido es el deber cumplir, el modelo a seguir, la norma a aplicar y la cual puede llegar a ajustar la conducta de los actores.

El discurso instituido pretende sujetar a una forma dominante de objetivar la realidad social, es decir, sujeta a una visión del mundo, a una manera de explicar y dar sentido al hacer, pensar y sentir. El discurso dominante intenta mantener esa forma de objetivar la realidad, pretende poner límites, dejar fuera otras formas de racionalizar u objetivar la realidad, pretende que este sistema de creencias no tenga fisuras. La narrativa y el discurso les permiten a las personas reconocerse, pero al mismo tiempo son formas de ideologización, homogeneización y sumisión. (2004, p. 107-109)

Teniendo en cuenta estos planteamientos, al hablar acerca del análisis del Discurso Instituido, se hace mención a la codificación y análisis de las actas de los consejos directivos y académicos, de los comités de calidad, los PEI y los manuales de calidad de las Instituciones Educativas vinculadas a esta investigación. Así entonces, el discurso instituido, es decir, el discurso escrito se evidenció en dos grandes categorías que son: el Discurso Administrativo, que se denominará DA, y el Discurso Pedagógico, denominado DP, cada uno de ellos conformado por unas subcategorías, el primero por las 4 áreas de gestión estipuladas en la guía 34 por el MEN, que son: $\mathrm{DA}_{-}$gestión administrativa y financiera, $\mathrm{DA}_{-}$ gestión directiva, DA_ gestión comunitaria y social y DA_ gestión académica. Y el segundo por: DP_ Los fundamentos y principios de los procesos educativos, $\mathrm{DP}_{-}$las interacciones entre actores educativos, $\mathrm{DP}_{-}$ los ambientes, contextos y escenarios educativos, $D_{-} P_{-}$los procesos y prácticas curriculares, $\mathrm{DP}_{-}$los procesos y prácticas didácticas, DP_ los procesos y prácticas de evaluación y por último DP_ los recursos y medios educativos. Cada una de estas subcategorías, contienen la cantidad de unidades semánticas (oraciones con sentido) que sustentan la incidencia de los SGC en las Instituciones Educativas de básica y media en el Distrito de Santa Marta. La figura 2 muestra la estructura de las categorías mencionadas.

En la Figura 3, se puede apreciar que en las Actas, PEI y Manuales de Calidad de las instituciones participantes hay una ligera mayor presencia de unidades semánticas propias de la categoría Discurso Administrativo, con 235 US, equivalentes al $51 \%$ del total, frente a 226 unidades semánticas (49\%) pertenecientes a la categoría Discurso Pedagógico. 


\section{- Figura 2.}

\section{Categorías del discurso instituido}

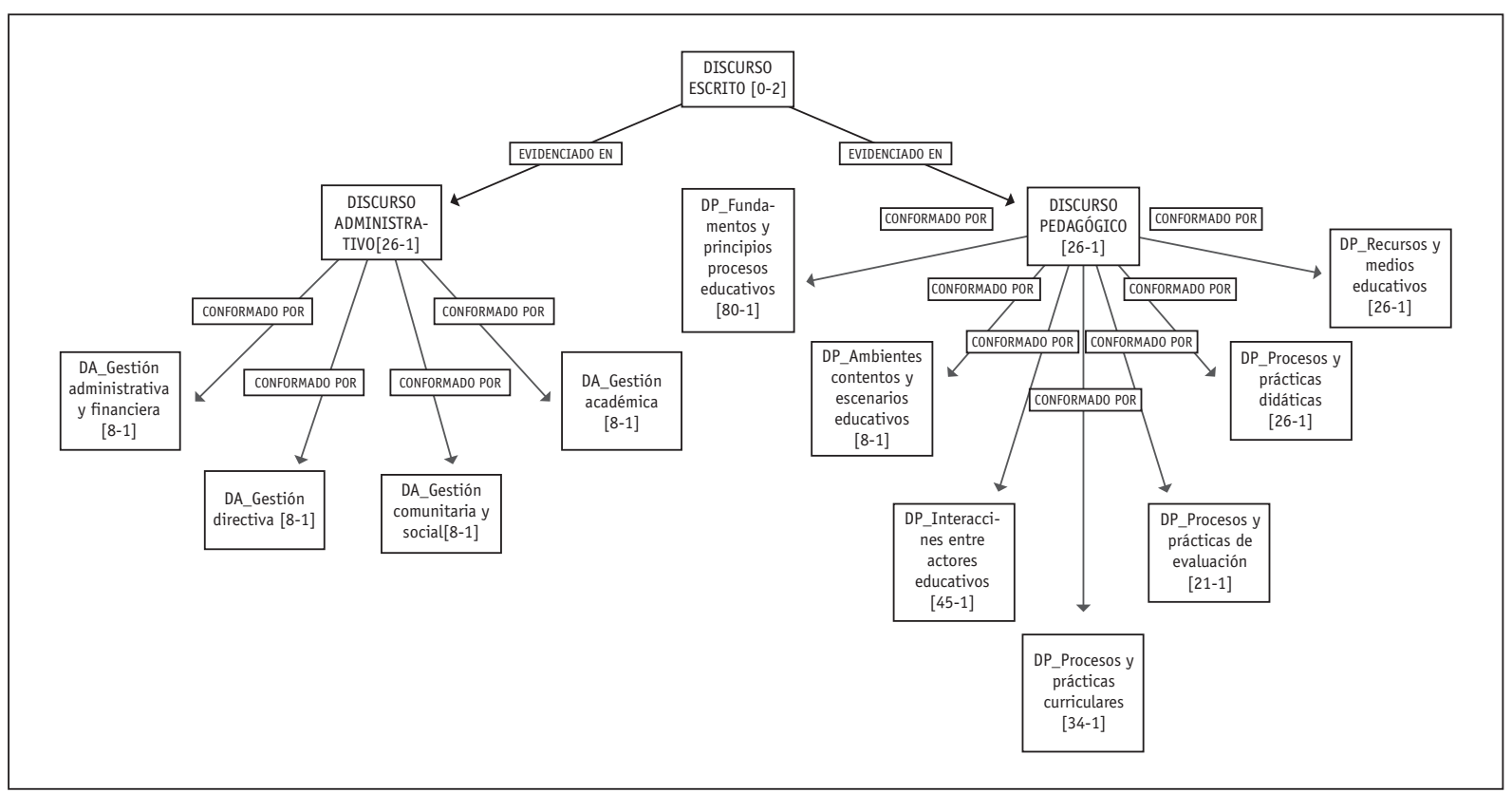

Fuente: Documentos (Actas, PEI, Manuales de calidad) y textos orales transcritos recopilados en talleres con grupos focales de discusión, y procesados en Software Atlas- ti.

\section{Figura 3.}

\section{Peso total discursos fuentes instituidas}

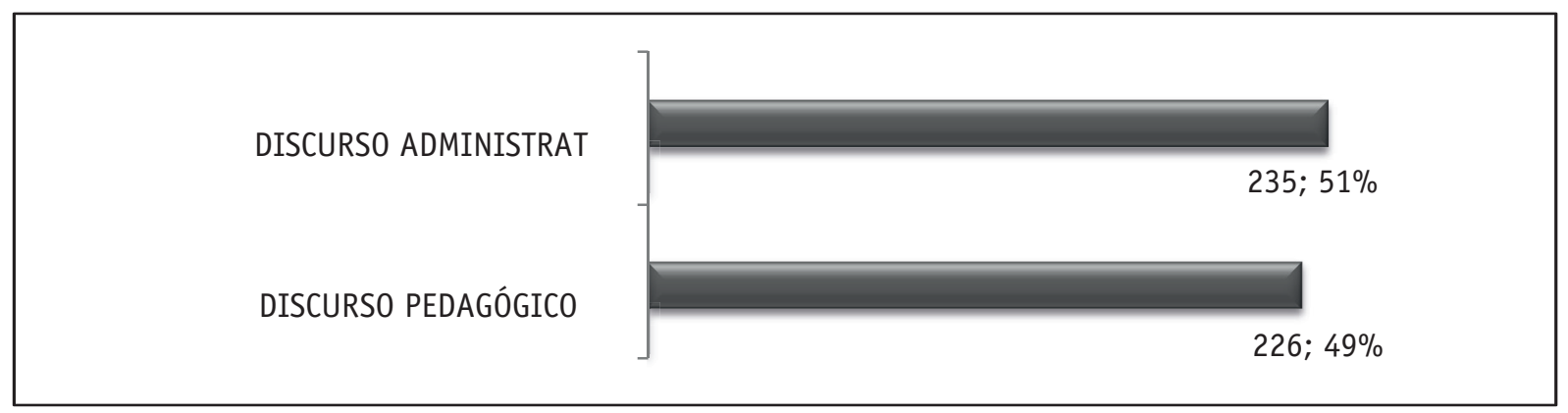

Fuente: Documentos (Actas, PEI, Manuales de calidad) procesados en Software Atlas- ti.

La Figura 3, muestra una relación relativamente nivelada, donde pareciese que la presencia de ambos discursos en los documentos escritos es pareja, sin embargo, cabe resaltar que el volumen de textos codificados relativos principalmente al discurso pedagógico era mayor, esto es, documentos como PEI, actas de comité académico superaban en volumen a documentos como manuales de calidad o actas de comité de calidad. Esto conduce a inferir con base en estos hallazgos, que la relación posiblemente no hubiese sido tan equilibrada de contar con un volumen proporcional de textos relativos a ambos 
discursos, inclinando la balanza aún más hacia el discurso administrativo, en las fuentes instituidas.

Siguiendo con el análisis, ahora profundizando en las unidades semánticas halladas en las fuentes instituidas propias del $D A$, se tiene que dentro de estas existe un numero de aspectos que presentan una mayor recurrencia, los cuales se presentan a continuación: El trabajo por procesos y procedimientos, la implementación de formatos, la recopilación de evidencias y soportes, la incursión de una nueva terminología y requisitos por cumplir. Tales aspectos se encuentran bastante claros, explícitos y referenciados en los PEI y Manuales de Calidad, como por ejemplo, en un manual de Calidad de una Institución certificada a través de la norma ISO se encontró el siguiente relato;

"Se ha documentado e implementado el Control de Servicio no conforme, el cual permite establecer el método para identificar y controlar el servicio no conforme detectado en el Proceso de Formación, con el fin de cumplir con los estándares de calidad del servicio educativo." (Manual de Calidad de institución focal 3)

Otro ejemplo de la presencia de aspectos propios del DA, es lo hallado en las actas de los comités de calidad, así entonces, en una de dichas actas se encontró: "Acerca de la determinación de los formatos que debe manejar el Comité para las diferentes clases de información y documentos se acordó estructurar los correspondientes a: oficios remisorios, acuerdos, protocolizaciones, POAS, actas, reglamentos, informes, encuestas, propuestas". (Acta Comité de Calidad de institución focal 1).

\section{Ocultamiento del discurso pedagógico en fuentes instituyentes}

Cuando se aborda el discurso en su dimensión instituyente, se hace referencia a todos aquellos aspectos innovadores que los actores vinculados a una organización proponen, ya sea como protesta 0 alternativa hacia lo que está instituido. En este sentido, Schvarstein, (1992) nombra a la organización como "sujeto", afirmando que lo instituyente no es una propiedad del objeto, sino que pertenece a la descripción del observador (citado en Belmonte \& Gadano, 2007, p. 116).

Esta dimensión del discurso se caracteriza por dar prioridad al punto de vista de los actores que participan en el proceso, brindando nuevas posibilidades o alternativas de aplicación de determinadas normas. González, (2004, p. 120) plantea que el discurso instituyente es aquel que busca abrir los límites de la normalidad para hacerse un espacio. Es aquel que tiene en cuenta el territorio de los otros. Cada significado discursivo pone en movimiento fuerzas contrarias que lo desestabilizan y generan conflicto y es esta relación entre el orden establecido y el conflicto donde se ponen las bases para comprender el devenir entre lo que persiste y el cambio.

Innovación, cambio, prioridad a los puntos de vista, pensamientos, sentimientos y actuaciones de los miembros que conforman la comunidad educativa, son los principales aspectos que se abordan en este tipo de discurso, evidenciando la capacidad que tienen las personas para articular su pensar, su ser y su actuar con la norma ya establecida. Teniendo en cuenta la pluralidad de cada ser humano, brindando así una posibilidad de adaptación, implementando prácticas concretas de lo establecido con aquellas necesidades que surjan en el devenir institucional.

Para fines de esta investigación, el discurso instituyente o discurso oral, se obtuvo después de la realización de los grupos focales de discusión y las entrevistas en profundidad dirigidas a rectores, coordinadores, líderes de calidad y docente en cada una de las tres Instituciones Educativas que cuentan con certificación por la Norma ISO o EFQM, vinculadas al proceso investigativo.

El análisis aquí hecho, al igual como se hizo con el discurso derivado de fuentes instituidas, tuvo en cuenta unas categorías orientadoras, tales como: Prácticas y Discurso pedagógico (PYDP), como en las Prácticas y Discurso Administrativo (PYDA). A la vez, estos discursos están conformados por unas subcategorías que ayudaron en la recopilación, procesamiento, análisis y comprensión: a nivel de las PYDA se encuentran: PYDA_gestión académica, PYDA_gestión administrativa y financiera, PYDA_ges- 
tión comunitaria y social y PYDA_gestión directiva. Por su parte, las PYDP agrupa a: PYDP_fundamentos y principios de los procesos educativos, PYDP_interacciones entre actores educativos, PYDP_ambientes, contextos y escenarios educativos, PYDP_procesos y prácticas curriculares, PYDP_procesos y prácticas didácticas, PYDP_procesos y prácticas de evaluación y por último PYDP_recursos y medios educativos, a partir de las cuales surgieron las unidades semánticas.

Anteriormente se mencionó, que las categorías propias del discurso administrativo tienen una mayor presencia de unidades semánticas en los documentos codificados (Actas, PEI y Manuales de Calidad); consecuentemente, en la dimensión instituyente es un poco más aguda la diferencia de pesos entre los discursos administrativos y pedagógicos, donde nuevamente el discurso administrativo se erige frente al pedagógico. En la figura 4. Se muestra la diferencia de pesos porcentuales entre los discursos, donde el administrativo se evidencia en la fuentes instituyentes con un 56\%. En relación a esto, los actores implicados en el proceso de certificación, manifiestan en sus intervenciones que las exigencias y requerimientos solicitados son mayores, lo que los lleva a involucrarse mayormente en los asuntos de orden administrativo. Lo cual se puede evidenciar en la siguiente US, en la cual además, se encuentra la presencia de uno de los aspectos más recurrentes ("Gestión documental") en los discursos de los participantes de la investigación.

"Tenemos los cambios en la gestión documental, obviamente ya se manejan aún más carpetas, más formatos, más procesos, yo no he estado aquí tantos años pero si he estado desde finales del año pasado hacia acá y los compañeros que llevan más tiempo que yo, me confiesan o me expresan que la gestión documental se ha incrementado mucho ya no es igual a otros años anteriores". (Docente de institución focal 1).

\section{- Figura 4.}

\section{Pesos de discursos fuentes instituyentes (orales)}

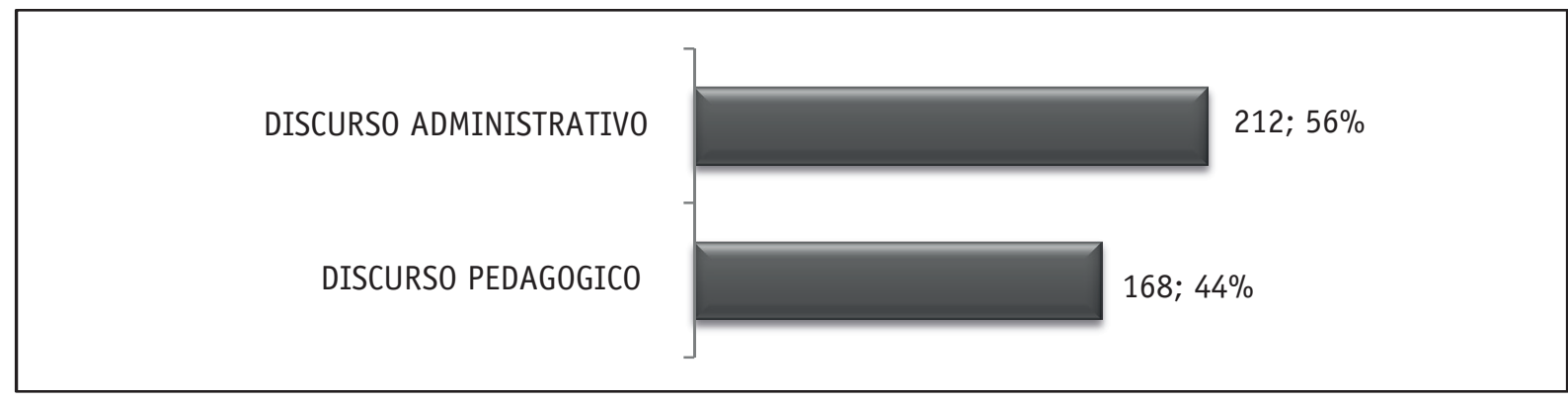

Fuente: Textos orales transcritos, recopilados en talleres con grupos focales de discusión, y procesados en Software Atlas- ti. 
En esta misma línea del análisis, se destaca la concepción recurrente que se tiene sobre los padres de familia, denominándolos a estos como "clientes" de los servicios educativos institucionales. La siguiente US se presenta como evidencia de lo afirmado:

"cambió la misión, la visión, el manual de convivencia, o sea todos los procesos del colegio entraron en un inicio de transición, entramos a evaluar, a conocer nosotros mismos la historia del colegio, a saber por qué tenía que cambiar, a conocer al padre de familia como un cliente, antes nosotros le hablamos al padre de familia totalmente diferente a la manera como se le está tratando ahora". (Coordinador de Calidad de institución focal 3)

\section{Conclusiones}

Esta investigación tuvo un firme propósito comprensivo, delimitado por dos vías, una de naturaleza teórica y otra empírica, en la cual se buscaba comprender las tensiones y transformaciones que ha suscitado la implementación de Sistemas de Gestión de la Calidad en las Instituciones Educativas, así como presentar evidencias que corroboraran o anularan la hipótesis sobre la desterritorialización del discurso pedagógico por parte del discurso administrativo en las instituciones educativas certificadas por algún modelo de gestión de la calidad. Para lo cual se hizo necesario el uso de una metodología cualitativa que brindara la posibilidad del alcance de los objetivos, así como la comprobación o nulidad de la hipótesis de la investigación. A continuación se presentan las conclusiones de la investigación.

Esta investigación planteó como hipótesis, que el Discurso pedagógico podría estar perdiendo terreno frente al discurso administrativo al interior de las Instituciones Educativas, debido a la llegada de los SGC a éstas. Luego de la revisión de los discursos, practicas discursivas y de los documentos oficiales de las instituciones (PEI, Manuales de Calidad, Actas de Comité directivo, académico, y de calidad) a través del Software "Atlas ti" Versión 6.0., mediante el cual se hizo un conteo de las Unidades semánticas (US) pertenecientes a cada uno de los discursos, se concluye que, efectivamente existe un cierto nivel de ocultamiento del discurso pedagógico tanto a nivel instituyente (Discursos y prácticas cotidianas al interior de las instituciones por parte de los actores) como en el nivel instituido (Documentos oficiales de la institución) frente al discurso administrativo. Los resultados muestran un total de 841 US encontradas tanto en fuentes instituidas como instituyentes, distribuidas entre las categorías discurso pedagógico y discurso administrativo, con 394 (47\%) y 447 (53\%) unidades semánticas respectivamente.

Esta ligera disparidad estuvo presente tanto en el análisis general, que incluye lo instituido y lo instituyente en un solo grupo de análisis, como en el análisis de fuentes instituidas y fuentes instituyentes por separado.

En este punto, a partir de los hallazgos encontrados, es necesario expresar, que los SGC, como estrategias y filosofías mediadoras hacia la calidad de las organizaciones, deben ser repensados por los organismos que los promueven, implementan y desarrollan, de tal manera, que cuando estos sistemas interpelen las realidades de las instituciones educativas, no pierdan de vista la naturaleza propia de estas organizaciones, ni las prácticas y concepciones que en las mismas se encuentran.

Los SGC están soportados sobre una robusta estructura conceptual y de prácticas administrativas, que debe ser objeto de revisión e integración a los contextos educativos, sin poner en riesgo las concepciones y prácticas educativas. La no supremacía de alguna de las lógicas discursivas sobre la otra, es lo que, en cierto modo, garantizaría la construcción de organizaciones altamente eficientes, eficaces y efectivas tanto en los aspectos de orden administrativos como en lo pedagógico. Es la tensión entre estas lógicas (administrativas y pedagógicas) la que posibilita y dinamiza el crecimiento integral de las IE. El desplazamiento u ocultamiento de uno de los discursos frente al otro pone en riesgo el desarrollo de una calidad integral al interior de las instituciones educativas.

Finalmente, es preciso expresar, que no se ha planteado que las IE deban renunciar a la posibilidad de implementar o seguir implementando sus propios SGC si no, que este tipo de sistemas deben ser repensados, armonizados y contextualizados desde la 
Ocultamiento del discurso pedagógico frente al discurso administrativo en la gestión de las organizaciones educativas

naturaleza de las realidades educativas, que integren su sentir, que sus impactos favorezcan el crecimiento de toda la organización sin ir en detrimento de lo que existe en las mismas: que el discurso pedagógico impregne y transforme las prácticas administrativas, explorando nuevas formas pedagógicas de ser y hacer desde lo administrativo, que el discurso y prácticas administrativas se nutran de los avances en pedagogía para que logre una mayor integración en las realidades de las IE.

\section{Referencias Bibliográficas}

Belmonte V. \& Gadano C. (2007). "Entre lo Instituido y lo Instituyente. La dimensión comunicacional de las Organizaciones". Revista de la Facultad de Derecho y Ciencias Sociales, Universidad Nacional del Comahue. 13, 113-126.

Bernstein, B. (2003). Hacia Una Sociología Del Discurso Pedagógico. Editorial Magisterio.
Deslauriers, J. (2004). "Investigación Cualitativa: Guía Práctica" ( 1 ed. RUDECOLOMBIA ed.). Pereira: Ediciones Papiro.

Ducrot, 0., \& Todorov, T. (1945). Diccionario enciclopédico de las ciencias del lenguaje. Siglo Ventiuno XXI.

Galicia, 0. (2009). "El Discurso Administrativo y la Tradición Pragmática". Obtenido de http://www.ejournal.unam.mx/ rca/229/RCA000022907.pdf

González, A. (2004) "Poder y discursos en la construcción social de las identidades docentes universitarias". Tesis doctoral Universidad de Valencia, facultad de filosofía y ciencias de la educación, departamento de didáctica y organización escolar.

Schvarstein, L. (2008). Nuevos Paradigmas para la Gestión Humana de las Organizaciones; Transcripción de la conferencia en el lanzamiento de la Especialización de Gestión Humana de las Organizaciones, Universidad Piloto de Colombia, Bogotá. Obtenido de http://www.contextosrevista.com.co/Revista\%202/A3_Nuevos_paradigmas.pdf 